\title{
Beam dynamics simulation of a double pass proton linear accelerator
}

\author{
Kilean Hwang* and Ji Qiang ${ }^{\dagger}$ \\ Lawrence Berkeley National Laboratory, 1 Cyclotron Road, Berkeley, California 94720, USA
}

(Received 6 December 2016; published 3 April 2017)

\begin{abstract}
A recirculating superconducting linear accelerator with the advantage of both straight and circular accelerator has been demonstrated with relativistic electron beams. The acceleration concept of a recirculating proton beam was recently proposed [J. Qiang, Nucl. Instrum. Methods Phys. Res., Sect. A 795, 77 (2015)] and is currently under study. In order to further support the concept, the beam dynamics study on a recirculating proton linear accelerator has to be carried out. In this paper, we study the feasibility of a two-pass recirculating proton linear accelerator through the direct numerical beam dynamics design optimization and the start-to-end simulation. This study shows that the two-pass simultaneous focusing without particle losses is attainable including fully $3 \mathrm{D}$ space-charge effects through the entire accelerator system.
\end{abstract}

DOI: 10.1103/PhysRevAccelBeams.20.040401

\section{INTRODUCTION}

A high power proton accelerator as a driver for production neutrinos and neutrons has important applications in basic science and the energy industry and is being actively studied around the world [1,2]. Superconducting radio frequency (rf) cavities provide a higher acceleration gradient with lower wall loss and a larger aperture, and thus are favorably adopted over normal conducting cavities for high power accelerators these days. Since the construction and operation costs of the superconducting cavities are expensive, the number of cavities are minimized through an optimal choice of cavity types for each energy band. In addition, by recirculating a beam to pass the cavities multiple times, the number of cavities can be made even less. The existing recirculating superconducting linear accelerators (LINAC) for electron beams provided the proof of concept not only for the cost efficiency but also for the beam quality conservation [3,4]. Moreover, the recirculating LINAC for lepton beams are actively considered by many accelerator facilities for upgrades or new machine proposals [5-7]. However, despite the success for an electron beam, the recirculating concept for a proton beam is not yet well studied. Especially, the subtlety of rf phase synchronization for a recirculated proton beam must be studied [8].

One of the main differences for the recirculating concept between the electron and proton beam stems from the longitudinal velocity which is nearly frozen for the electron

\footnotetext{
*kilean@lbl.gov

jqiang@lbl.gov
}

Published by the American Physical Society under the terms of the Creative Commons Attribution 4.0 International license. Further distribution of this work must maintain attribution to the author(s) and the published article's title, journal citation, and DOI. beam while it varies comparatively faster for the proton beam through high gradient superconducting rf cavities. This fact requires stronger restriction on rf phase synchronization for a medium energy proton beam. For example, considering the 150 and $300 \mathrm{MeV}$ proton beams, after only $70 \mathrm{~cm}$ in distance, there will be one rf cycle phase slippage between the two beams when the rf is $650 \mathrm{MHz}$.

When the beam passes LINAC only twice, the phase synchronization can be achieved with proper choice of the separation length between the cavities [8]. It can be understood by the following condition:

$$
L\left(1 / v_{1}-1 / v_{2}\right)=n T_{\mathrm{RF}}
$$

where $v_{1}$ and $v_{2}$ are the longitudinal beam velocities of the first and second passes, respectively, $L$ is the separation length, $T_{\mathrm{rf}}$ is the rf period, and $n$ is the integer multiple. Note that the separation length becomes larger and larger as the beam energy increases. In this configuration, the focusing channel is hard to be made of periodic cells. The nonperiodic structure makes the design of the focusing channel more challenging.

Another difference between the electron beam and the proton beam is the stronger space-charge force for a proton beam due to the lower relativistic factor $\gamma$. Especially, the achromatic condition of the arcs can be easily violated by the space-charge induced dispersion [9]. It is also important to check whether the stronger space-charge effects will cause a large beam halo formation in the nonperiodic LINAC structure.

The purpose of this paper is to assess the feasibility of the nonperiodic focusing LINAC channel of two energy beams and the potential impact of the space-charge effects on the proton beam distribution using self-consistent multiparticle simulation. The organization of the paper is as follows: After the introduction, we present the basic machine layout 


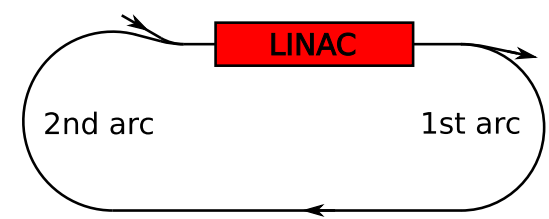

FIG. 1. Layout of the double-pass scheme.

in Sec. II, the beam dynamics design and simulation of the superconducting LINAC in Sec. III, the simulation of the recirculating arcs in Sec. IV, the start-to-end simulation of the entire double-pass system in Sec. V, and we draw some conclusions in Sec. VI.

\section{MACHINE LAYOUT}

A multi-GeV recirculating proton LINAC was proposed in Ref. [8]. There, an initial $150 \mathrm{MeV}$ proton beam is accelerated in three sections of recirculating LINAC to attain a final $8 \mathrm{GeV}$ energy. In this paper, we focus on the first section of the recirculating LINAC which is a doublepass recirculating LINAC accelerating the beam from 150 to $500 \mathrm{MeV}$. The basic machine layout is shown in Fig. 1 schematically. It consists of a section of a straight superconducting LINAC, two arcs, and a straight transport/ bunching line. The first pass of LINAC accelerates the beam from 150 to $306 \mathrm{MeV}$. The second pass of LINAC accelerates the beam from 306 to $500 \mathrm{MeV}$. The synchronous condition Eq. (1) is satisfied by adjusting the separation between two cavities so that the low energy beam in the first pass and the high energy beam in the second pass will have the same RF design phase [10]. Some major parameters used in this study are shown in Table I and are similar to Project- $X$ design parameters where 5-cell elliptical superconducting rf cavities are used for a 177$480 \mathrm{MeV}$ energy band [11]. Here, we have used two bunch currents in the beam dynamics simulation to study the potential space-charge effects.

\section{THE SUPERCONDUCTING LINAC}

The striaght rf LINAC section consists of 17 superconducting rf cavities and the separation length between the

TABLE I. Parameters used for double-pass proton LINAC design. Bunch current represent the average current over a single rf bucket.

\begin{tabular}{lc}
\hline \hline Item & Value \\
\hline Bunch current & $20,40 \mathrm{~mA}$ \\
Normalized transverse emittance & $0.23 \mathrm{~mm} \mathrm{mrad}$ \\
Normalized longitudinal emittance & $3.0^{\circ} \mathrm{MeV}$ \\
Radio frequency & $650 \mathrm{MHz}$ \\
Radio-frequency geometric beta $\beta_{G}$ & 0.63 \\
Accelerating gradient & $14 \mathrm{MV} / \mathrm{m}$ \\
Radio-frequency phase & $-30^{\circ}$ \\
\hline \hline
\end{tabular}

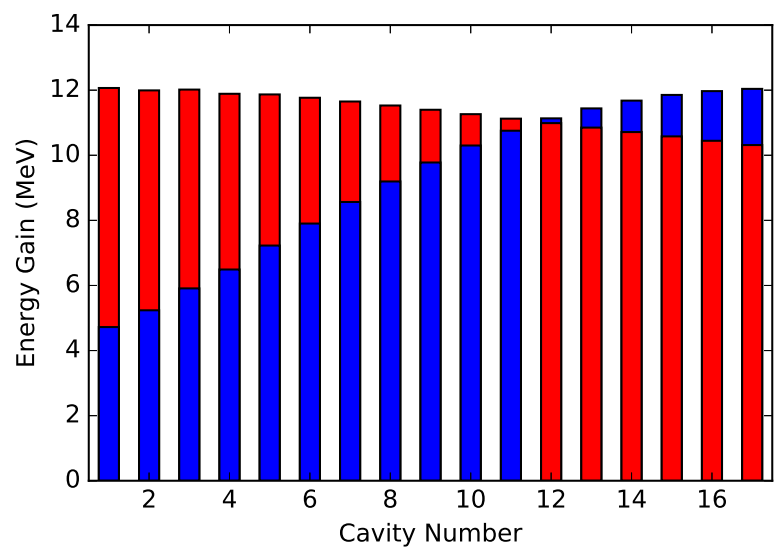

FIG. 2. Energy gain along the LINAC. Blue is the first pass and red is the second pass.

cavities are chosen according to Eq. (1) The energy gain along the LINAC for both passes is shown in Fig. 2 Passing the same structure twice, the beam is accelerated from 150 to $500 \mathrm{MeV}$. All the rf phases, except the second and the fouth cavities, are synchronized to $-30^{\circ}$ away from the maximum acceleration phase. In order to overcome the space-charge and rf defocusing and lack of the space for quadrupoles, we set the rf phases of the second and the fourth cavities as $+30^{\circ}$ to provide transverse rf focusing in these two cavities [12].

Figure 3 shows the separation length between cavities following Eq. (1) with $n=1$. The separation between two rf cavities increases as the proton beam energy increases. Compared to the periodic lattice structure, the nonperiodic lattice design is more challenging. Here, we rely on numerical optimization to design the nonperiodic focusing channel which has to focus the two beam passes with different energy simultaneously. We used the particle-incell code IMPACT [13] for tracking and the differential evolution algorithm [14] for global optimization. The cost function is a weighted summation of the beam rms

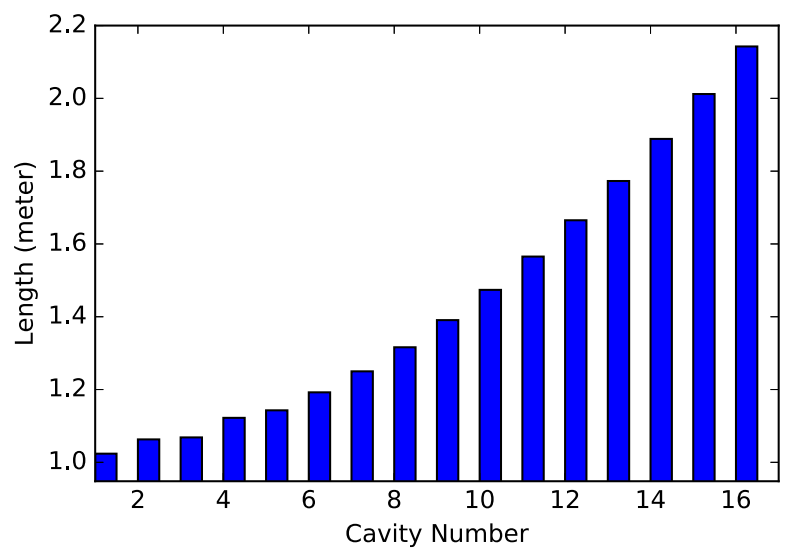

FIG. 3. Separation length from a cavity entrance to the next cavity entrance. 

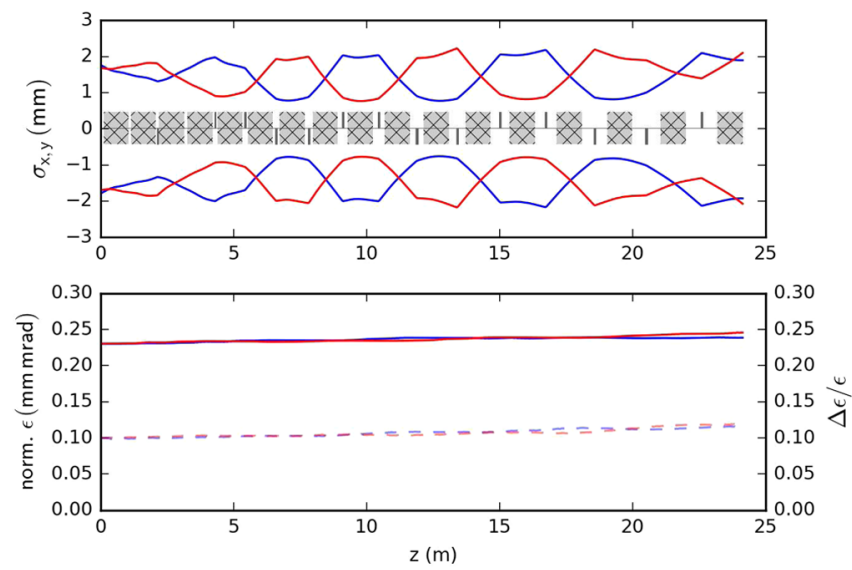

FIG. 4. rms beam envelope for the first pass using 6D waterbag initial distribution with $20 \mathrm{~mA}$ bunch current. Radio-frequency cavities are represented by " $x$ " patterned boxes, and the F/D quadrupoles are represented by up/down rods. The blue/red curves corresponds to horizontal/vertical. The dashed curve represents $\Delta \epsilon / \epsilon$.

envelope oscillation along the LINAC and normalized emittance growth at the exit of the LINAC,

$$
\begin{aligned}
\operatorname{cost}= & w_{\sigma} \sum_{i=0}^{N}\left\{\left[\sigma_{x}(i)-\sigma\right]^{4}+\left[\sigma_{y}(i)-\sigma\right]^{4}\right\} \\
& +w_{\epsilon} \sum_{i=x, y, z}\left[\epsilon_{i}(L)-\epsilon_{i}(0)\right]^{2}
\end{aligned}
$$

where $w_{i}$ are weights (also normalization constant) of the envelope oscillation contribution, $\sigma$ is the desired average root mean square (rms) beam size which is set to $1.5 \mathrm{~mm}, N$ is the number of integration step, and $L$ is the LINAC length. The $6 \mathrm{D}$ waterbag initial distribution was used during the optimization so that the resulting focusing channel is insensitive to the tail distribution of the beam [15]. We optimize the lattice with two different bunch currents 20 and $40 \mathrm{~mA}$. Figures 4 and 5 show the resulting
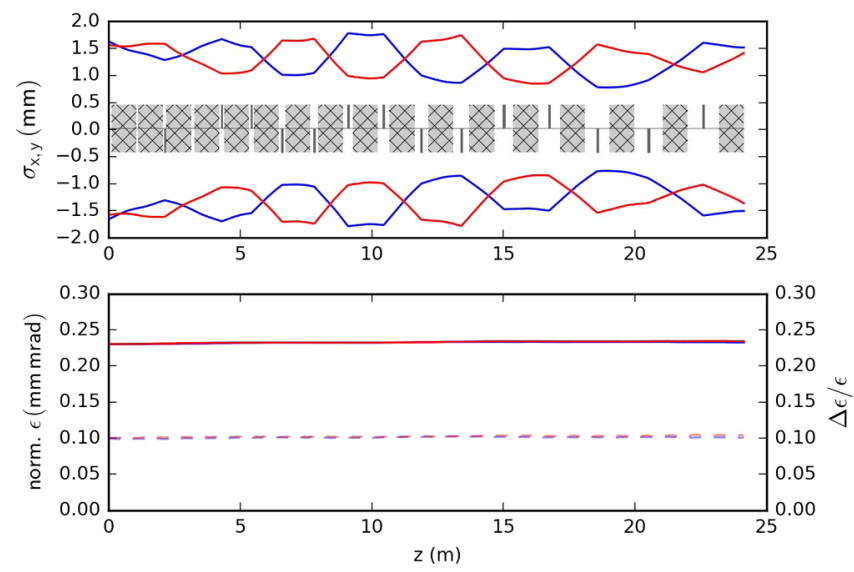

FIG. 5. rms beam envelope for the second pass using 6D waterbag initial distribution with $20 \mathrm{~mA}$ bunch current.
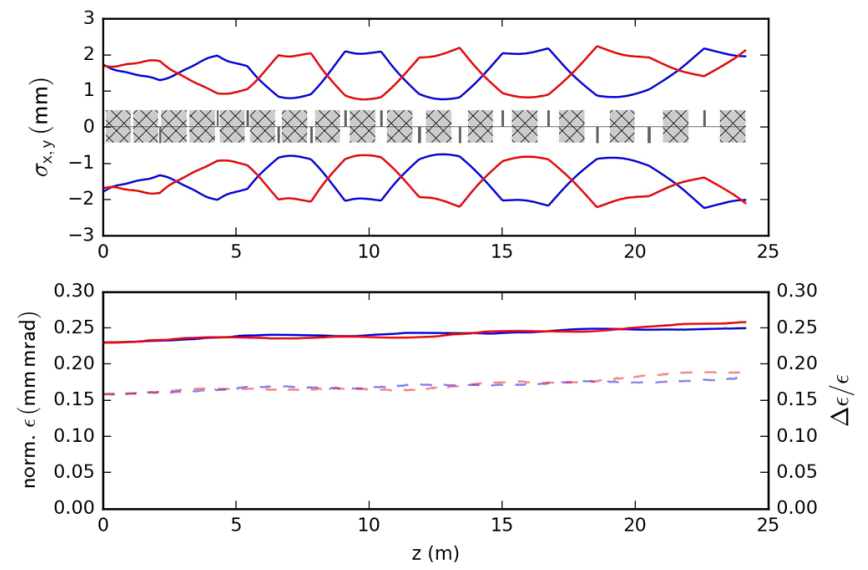

FIG. 6. rms beam envelope for the first pass using 6D Gaussian initial distribution with $20 \mathrm{~mA}$ bunch current.

rms beam envelope of the first and second passes, respectively, on top of the optimized focusing channel for the case of a $20 \mathrm{~mA}$ bunch current. The optimization resulted in a focusing-focusing-defocusing-defocusing (FFDD) lattice which makes the lattice look periodic with each cell containing four cavities. However, some of these cells do not even have a periodic optics solution. Nevertheless, since the beam optics on these cells are optimized using a full 3D particle-in-cell tracking code, the beam is stable throughout the structure. The dashed line corresponds to $\Delta \epsilon \equiv 1-\epsilon_{95} / \epsilon$ where $\epsilon_{95}$ represents the normalized rms emittance of the $95 \%$ inner most particles of the CourantSnyder circle [16]. The growth of $\Delta \epsilon / \epsilon$ can be interpreted as a measure of the tail distribution diffusion. We observe the rms beam envelope is well optimized smoothly for both passes and the normalized emittance growth is negligible. Figures 6 and 7 present the simulation of the first and second pass, respectively, using 6D Gaussian initial distribution on the same structure. Both the rms envelope and the normalized emittance growth are similar to the waterbag distribution case. The nonlinear space charge and the
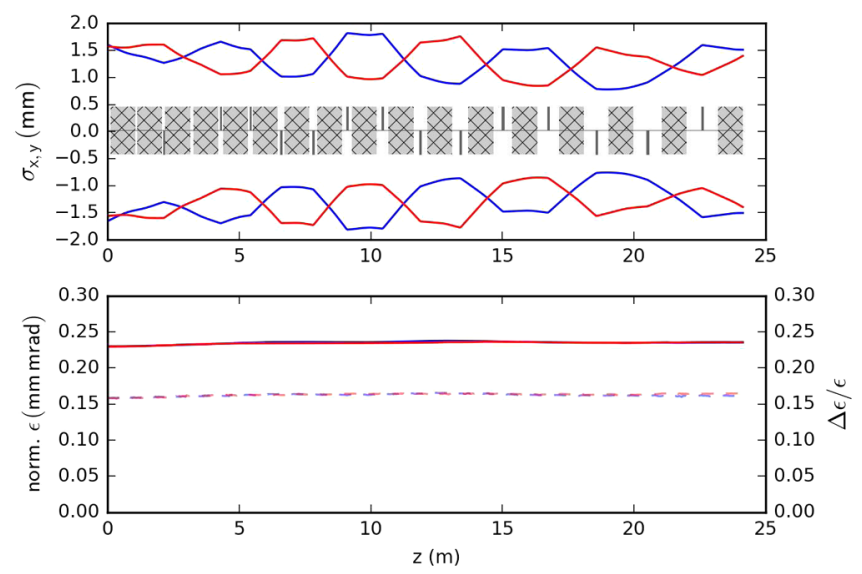

FIG. 7. rms beam envelope for the second pass using 6D Gaussian initial distribution with $20 \mathrm{~mA}$ bunch current. 

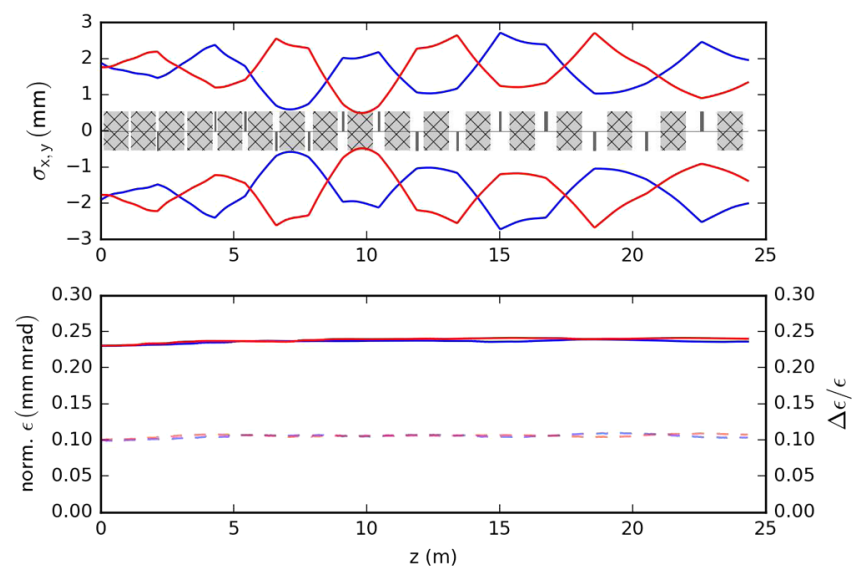

FIG. 8. rms beam envelope for the first pass using 6D waterbag initial distribution with $40 \mathrm{~mA}$ bunch current.
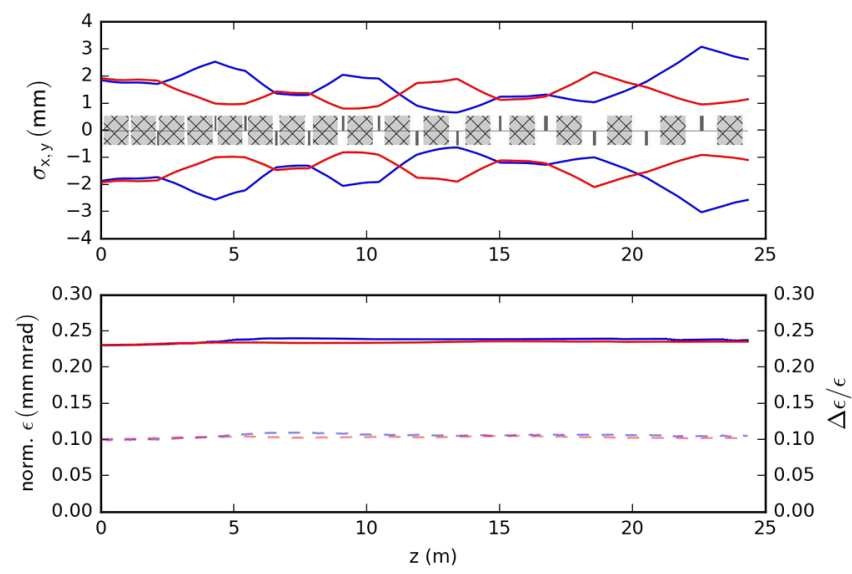

FIG. 9. rms beam envelope for the second pass using 6D waterbag initial distribution with $40 \mathrm{~mA}$ bunch current.
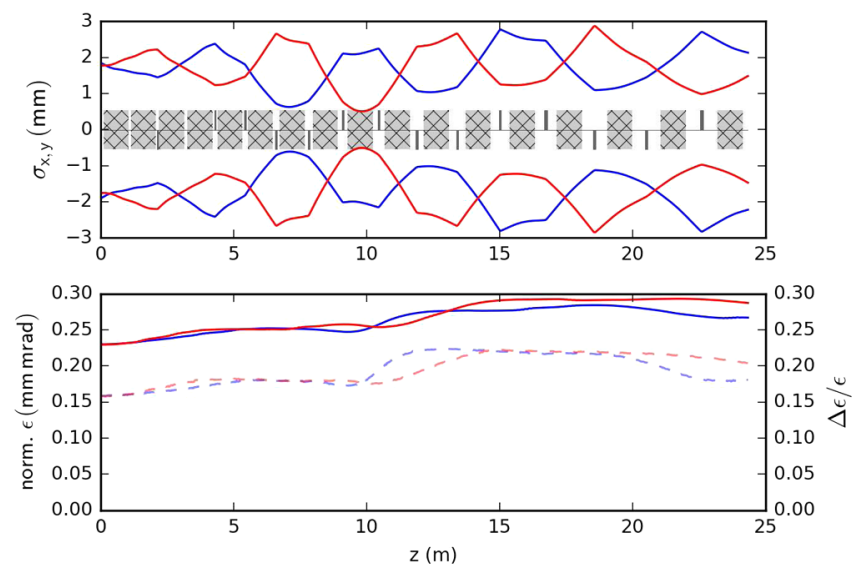

FIG. 10. rms beam envelope for the first pass using 6D Gaussian initial distribution with $40 \mathrm{~mA}$ bunch current.
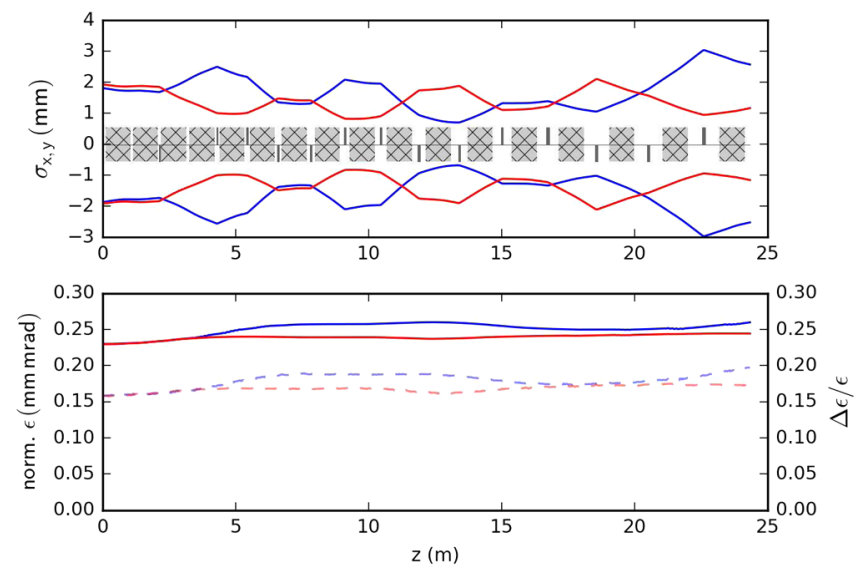

FIG. 11. rms beam envelope for the second pass using 6D Gaussian initial distribution with $40 \mathrm{~mA}$ bunch current.

tail from the Gaussian distribution do not cause significant extra envelope and emittance growth.

Figures $8-11$ show the optimization result with a $40 \mathrm{~mA}$ bunch current. In order to reduce computational load, we constrained the upper and lower bounds of the quadrupole knobs around the already obtained solution of the $20 \mathrm{~mA}$ case. Compared to Figs. 4-7, one can see that the envelope is not as smooth as the $20 \mathrm{~mA}$ case. This might be ascribed to the localized optimization knobs. There also is a slightly larger normalized emittance growth for the Gaussian initial distribution. However, such an onset of emittance growth tends to be stabilized downstream as shown in the start-toend simulation in Fig. 20.

\section{THE RECIRCULATING ARCS}

The two-pass recirculating proton LINAC studied here consists of two $180^{\circ}$ arcs to bend the beam back for the second-time acceleration. The first arc at the exit of the LINAC consists of five bending magnets where the first
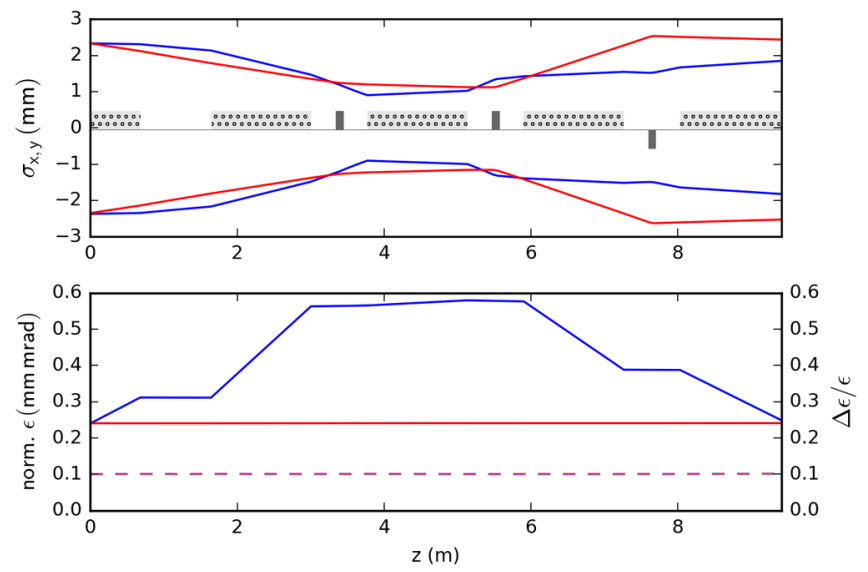

FIG. 12. rms beam envelope through the first arc with waterbag initial distribution and $20 \mathrm{~mA}$ bunch current. The circle patterned boxes represent the bending magnets. 

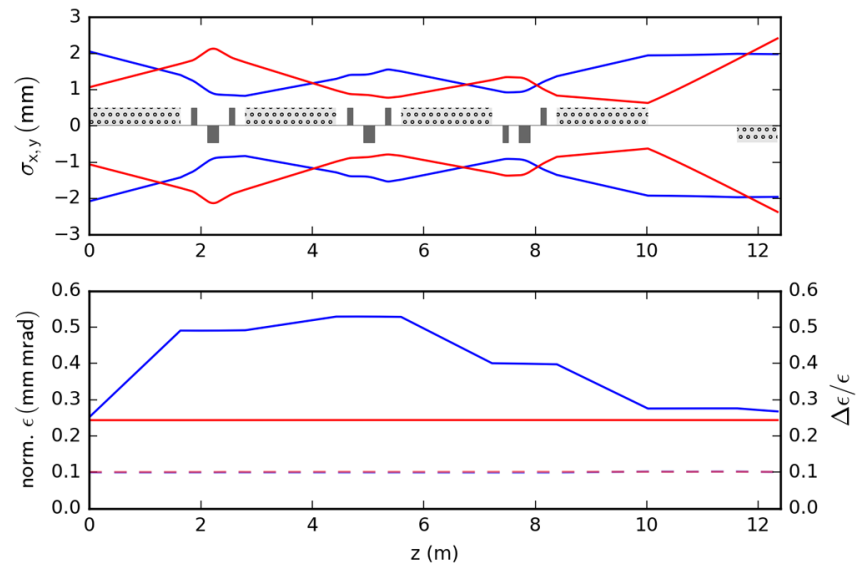

FIG. 13. rms beam envelope through the second arc with waterbag initial distribution and $20 \mathrm{~mA}$ bunch current. The downward circle patterned box represent the bending magnet of negative bending angle.
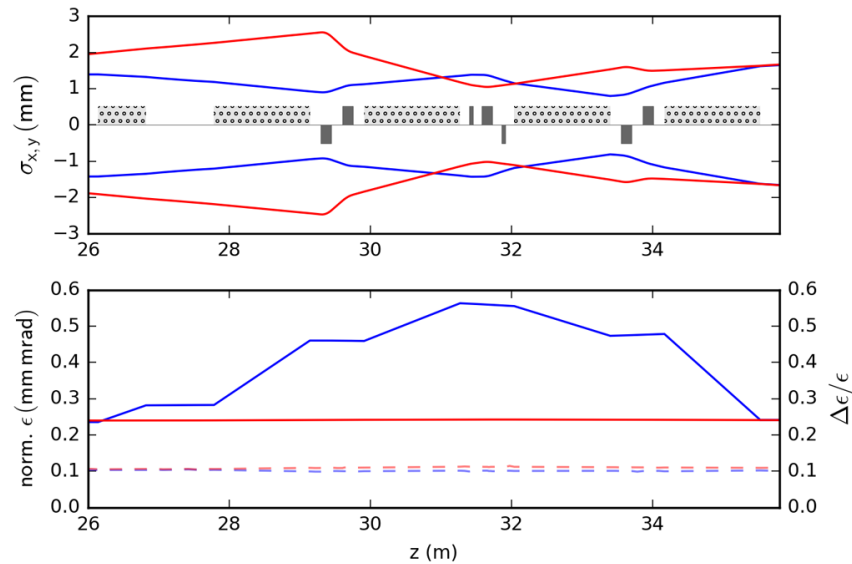

FIG. 14. rms beam envelope through the first arc with waterbag initial distribution and $40 \mathrm{~mA}$ bunch current.
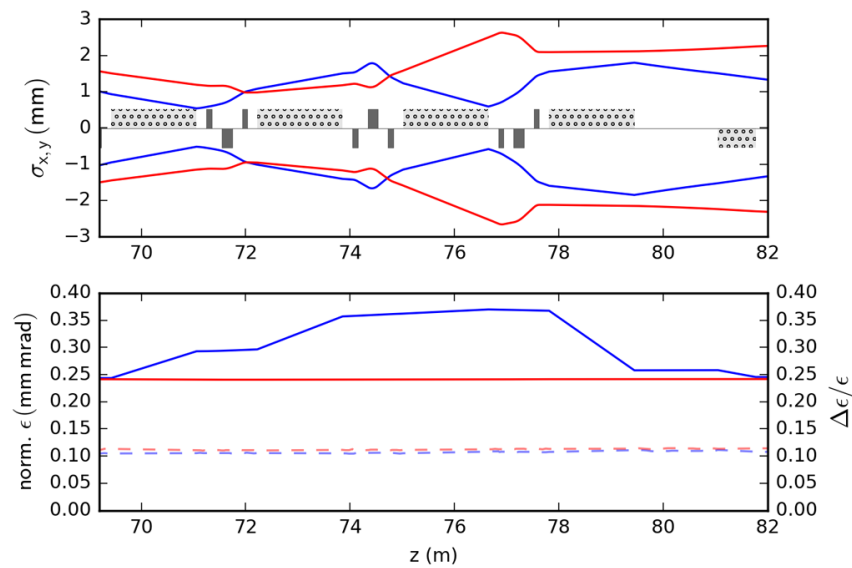

FIG. 15. rms beam envelope through the second arc with waterbag initial distribution and $40 \mathrm{~mA}$ bunch current.

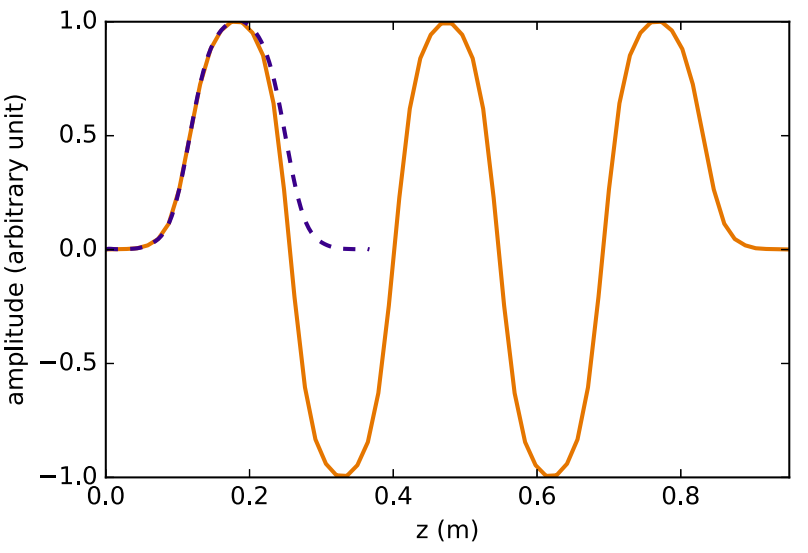

FIG. 16. Field profile of rf cavity. Orange line represents the 5 cell rf cavity used for Project-X. Blue dashed line represents the single cell bunching cavity.

one is used for splitting the two different beam energies. The second arc for the reentry to the LINAC also consists of five bending magnets where the bending angle of the last one is negative for merging the two different beam
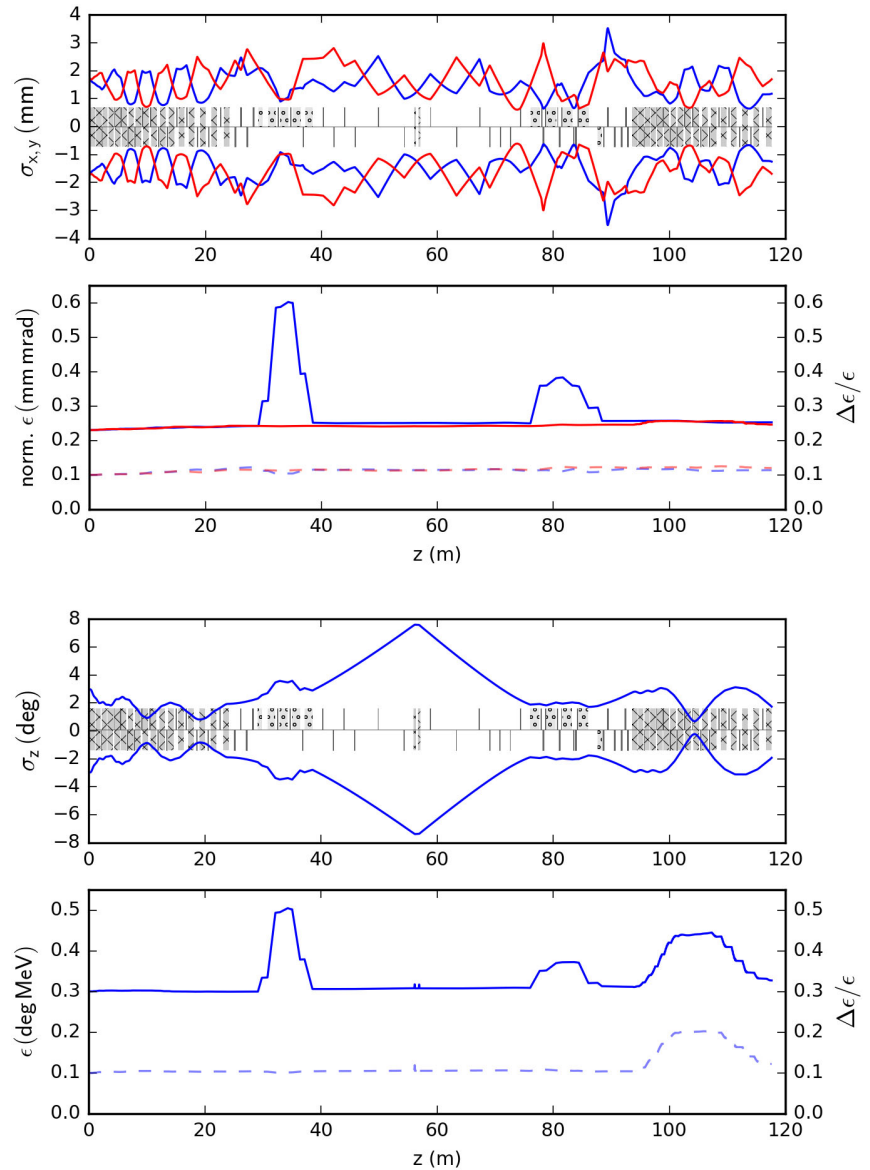

FIG. 17. rms beam statistics from the first-pass LINAC entrance to the second-pass LINAC exit with $20 \mathrm{~mA}$ bunch current and a waterbag initial distribution. 
energies. One beam dynamics concern through those arcs is the achromatic condition under the space-charge influence. Figure 12 shows the arc layout and the beam rms envelope and emittance evolution through the first left arc for the $20 \mathrm{~mA}$ bunch current case. It is a simple achromat with single quads between the dipoles. No quadrupole is introduced between the splitting dipole and the adjacent dipole. Little emittance growth is observed through this arc. However such a simple layout did not work for the second arc which is longer and includes a negative bending angle dipole for merging as shown in Fig. 1.

In addition to the zero-current achromatic condition, the following conditions can be used to reduce the spacecharge induced dispersion [9]:

$$
\begin{aligned}
& \int_{-L / 2}^{L / 2} \frac{M_{12}(z)}{\rho(z)} z d z=0, \\
& \int_{-L / 2}^{L / 2} \frac{M_{11}(z)}{\rho(z)} z d z=0,
\end{aligned}
$$

where $L$ is the length of the arc, $\rho$ is the bending radius, and $M_{i j}$ is the horizontal transport matrix map. Depending on
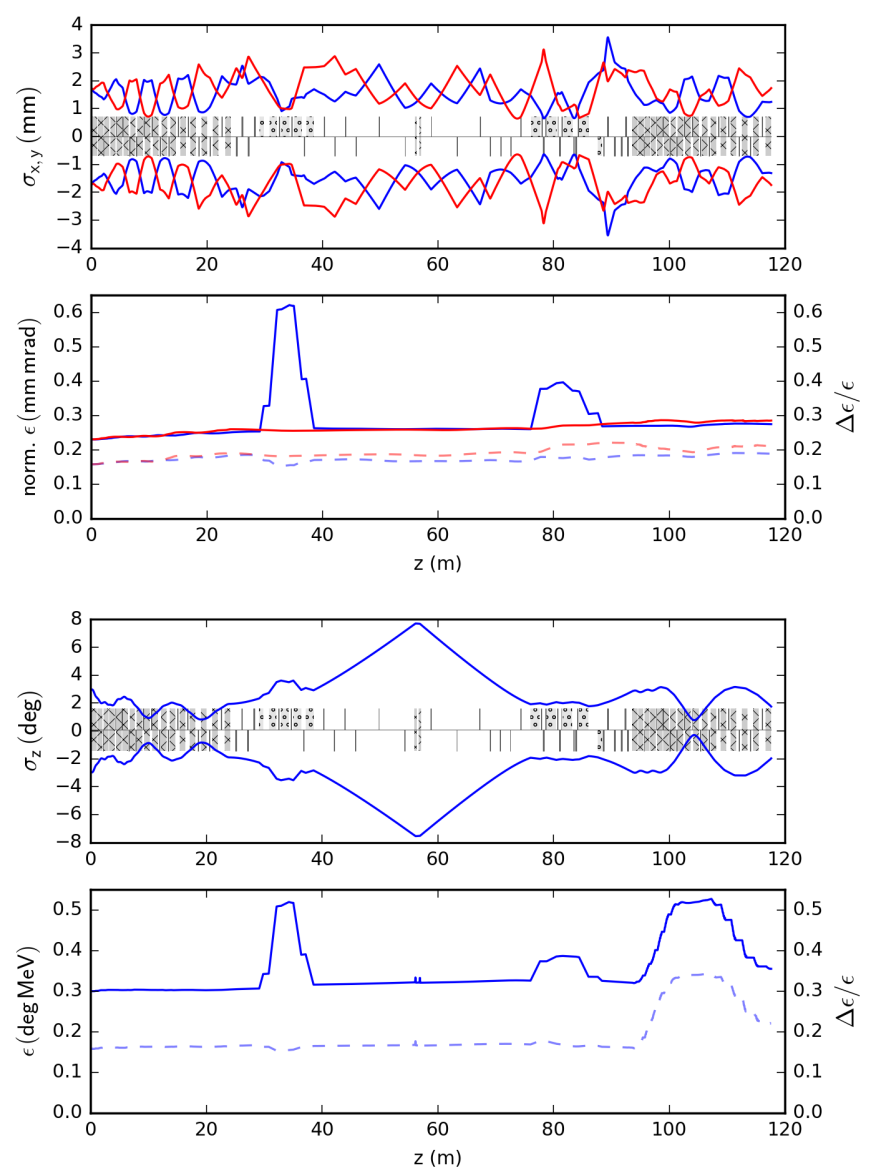

FIG. 18. rms beam statistics from the first-pass LINAC entrance to the second-pass LINAC exit with $20 \mathrm{~mA}$ bunch current and a Gaussian initial distribution. lattice geometry, the exact solution for Eqs. (3), (4) may not exist. Instead, we collect the solutions of the quadrupole control knobs that produces transfer matrix satisfying Eqs. (3), (4) as much as possible in addition to the zero current achromatic condition. Then, we optimize the initial optics functions which define initial beam distribution for each solution. The selected solution for the second arc is shown in Fig. 13 in the case of a $20 \mathrm{~mA}$ bunch current. The growth of $\Delta \epsilon / \epsilon$ is negligible with this current.

In the case of a $40 \mathrm{~mA}$ bunch current, we observed an emittance increase of about $15 \%$ through the second arc with a waterbag initial distribution. So we took a different route to redesign the arcs for the $40 \mathrm{~mA}$ case. The chromaticity, and the space-charge induced chromaticity, have to be reoptimized as well as the dispersion and the space-charge induced dispersion. Instead of introducing sextupoles for chromatic correction, we optimized quadrupole knobs with limited space of magnets for compact arcs according to Eq. (2). Figures 14 and 15 show the results for the first and the second arc, respectively, with a $40 \mathrm{~mA}$ bunch current. The emittance growth is negligible after the reoptimization for the $40 \mathrm{~mA}$ current case.
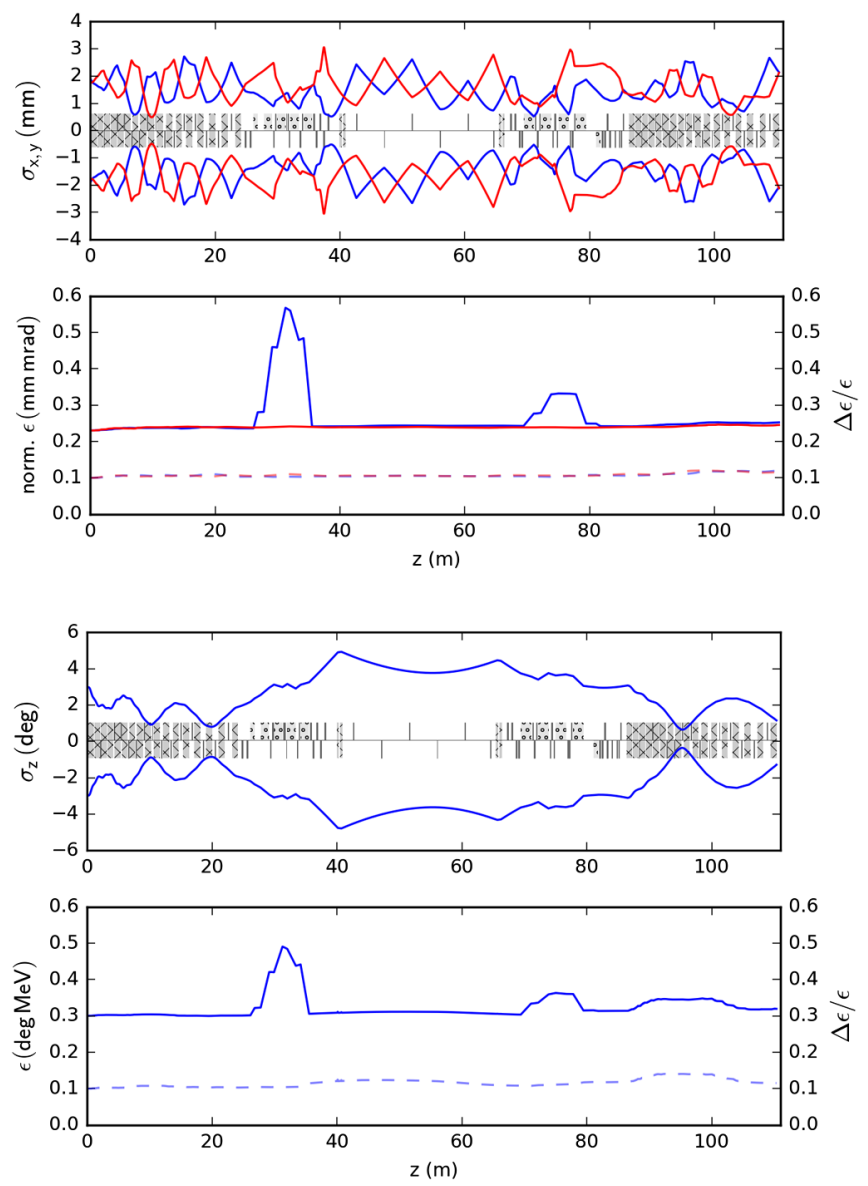

FIG. 19. rms beam statistics from the first-pass LINAC entrance to the second-pass LINAC exit for $40 \mathrm{~mA}$ bunch current and waterbag initial distribution. 


\section{START-TO-END BEAM DYNAMICS SIMULATION}

Outside the superconducting LINAC without rf focusing, the proton beam will start to lengthen due to the spacecharge force and the finite longitudinal emittance. In order to keep the beam bunched throughout the recirculation path, bunching cavities are introduced at the middle of long straight section with $5.2 \mathrm{MV} / \mathrm{m}$ acceleration gradient for the $20 \mathrm{~mA}$ case and at the beginning and the ending of the long straight section with $7.7 \mathrm{MV} / \mathrm{m}$ and $5.1 \mathrm{MV} / \mathrm{m}$ acceleration gradient, respectively, for the $40 \mathrm{~mA}$ case. We concatenated the field profile of the first and last half cell of the LINAC cavity to assume the field profile of the bunching cavity as shown in Fig. 16. Two of such cavities are used at each bunching section. The same rf frequency as the LINAC cavities is used and the rf phase is fixed at $-90^{\circ}$ away from the maximum acceleration phase. The length of the matching sections between LINAC and arc is reduced for the $40 \mathrm{~mA}$ case to further alleviate the bunch lengthening. One million macro particles and 128-128-128 3D Cartesian meshes are used through Figs. 17-22. Figure 17 shows the start-to-end beam dynamics simulation result
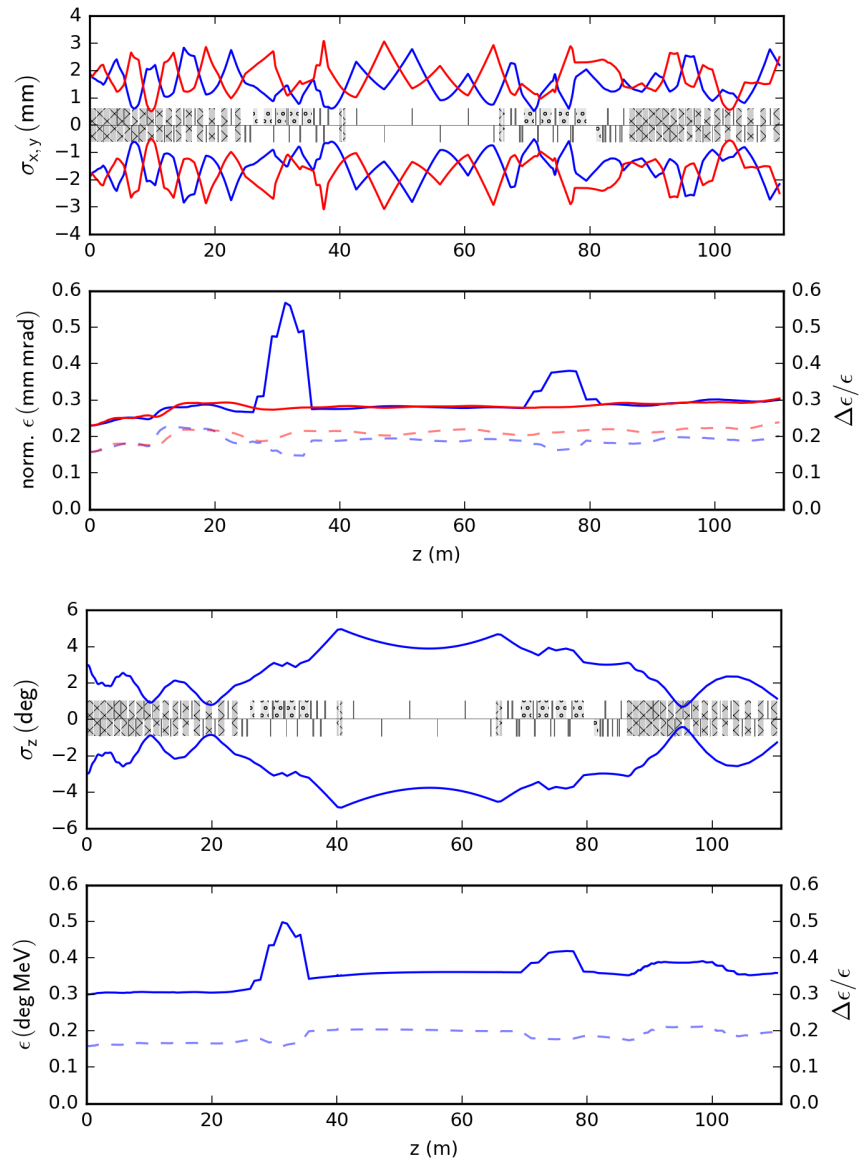

FIG. 20. rms beam statistics from the first-pass LINAC entrance to the second-pass LINAC exit for $40 \mathrm{~mA}$ bunch current and Gaussian initial distribution. from entrance to superconducting LINAC in the first pass to the exit of the LINAC after the second pass using the waterbag initial distribution with the current of $20 \mathrm{~mA}$. Start-to-end optimization was performed using the upper and lower bound around the already obtained solution from section-by-section optimization. The envelope at the second pass is not as smooth as it was before the start-to-end optimization but the normalized emittance growth is negligible. Figure 18 shows the simulation using Gaussian initial distribution where small normalized emittance growth is observed. Figures 19 and 20 show the simulation results in the case of a $40 \mathrm{~mA}$ beam current. The onset of the normalized emittance growth is seen for the Gausssian initial distribution while it is negligible for the waterbag initial distribution.

In all cases, one can see the longitudinal emittance growth through the second pass of the superconducting LINAC. After investigation of the longitudinal phase space, we found that such a growth is due to longitudinal phasespace mismatch. However, this is not a limiting factor for a recirculating proton LINAC. Recall that we have synchronized most of the rf cavity phases simply to $-30^{\circ}$ in this
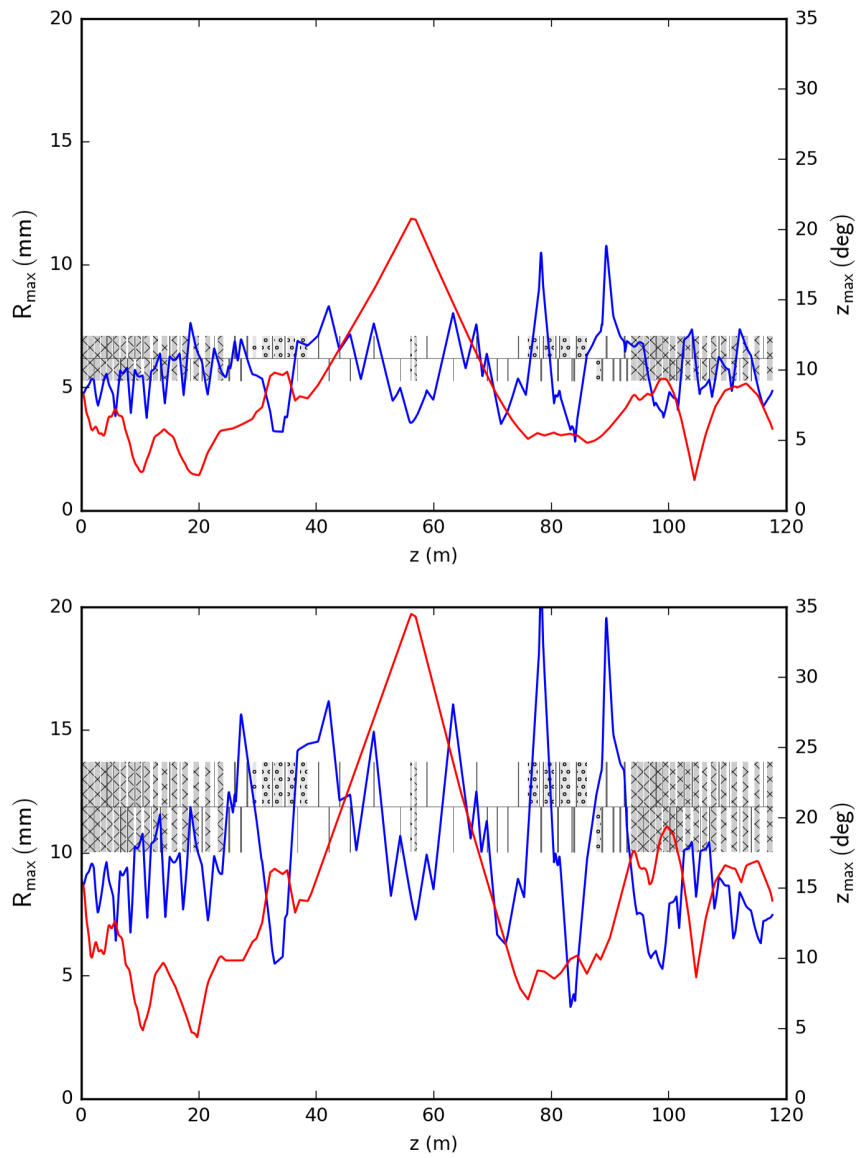

FIG. 21. Maximum transverse radius (blue) and longitudinal (red) phase for $20 \mathrm{~mA}$ case with waterbag (top) and Gaussian (bottom) initial distribution. 

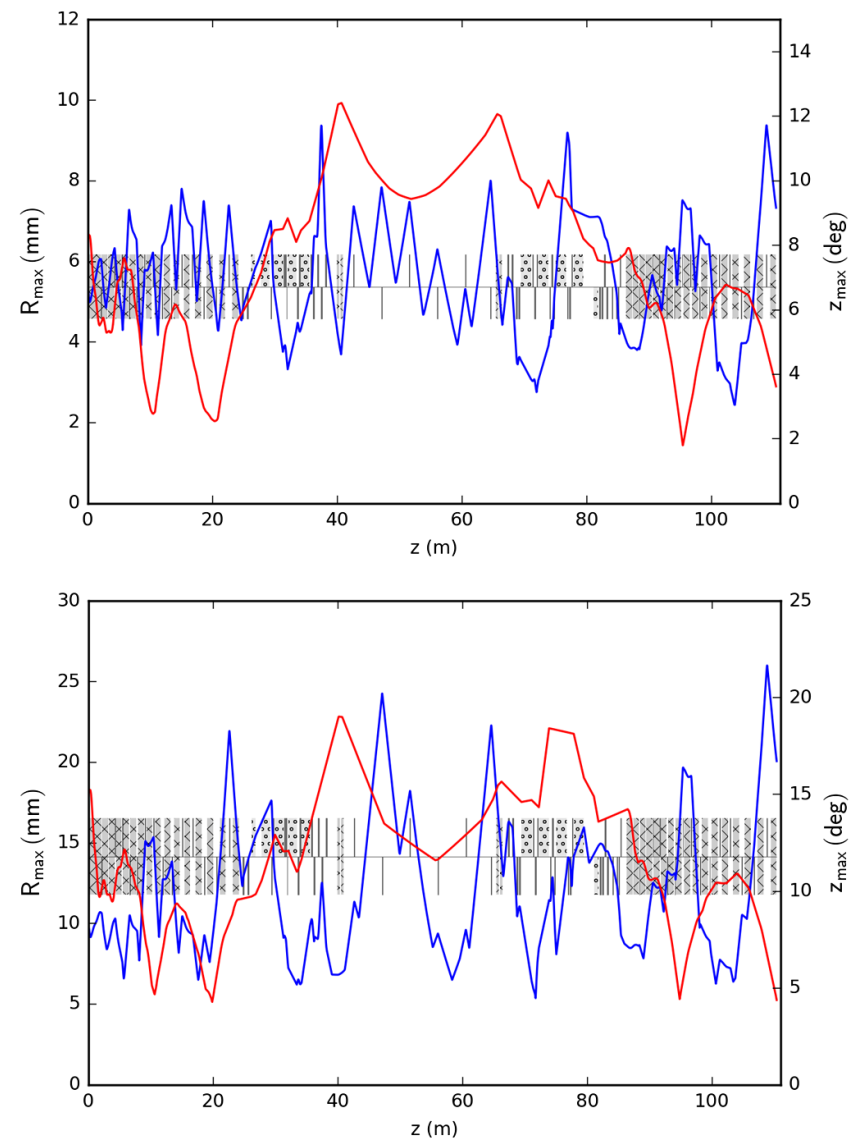

FIG. 22. Maximum transverse radius (blue) and longitudinal (red) phase for $40 \mathrm{~mA}$ case with waterbag (top) and Gaussian (bottom) initial distribution.

conceptual design. Further phase optimization can be made to match longitudinal optics.

Finally, Figs. 21 and 22 show the maximum transverse and longitudinal oscillation amplitudes evolution from the start-to-end simulation with the $20 \mathrm{~mA}$ and $40 \mathrm{~mA}$ beam current, respectively. The maximum transverse radius for both currents is below $2 \mathrm{~cm}$ through the superconducting cavities, which has an aperture diameter of $8.3 \mathrm{~cm}$ [11]. There will be no particle losses throughout the entire twopass accelerator system.

\section{CONCLUSION}

As a part of the feasibility study for a recirculating proton LINAC, a beam dynamics study has been carried out through the conceptual double-pass proton LINAC design. The simultaneous focusing in a nonperiodic structure during two passes of the proton beam through the superconducting LINAC was achieved. The space-charge effect through the recirculating arcs and straight transport line can be properly managed. The transverse beam size and the longitudinal bunch length are well below the aperture limit. There is no particle loss through the entire two-pass system from the start-to-end simulation. This provides better confidence for the recirculating proton LINAC concept.

It is also important to mention the possible limitations of a recirculating proton LINAC. First, when only two passes are considered the separation length between cavities become too short (long) for low (high) energy beams. This problem can be partially solved by allowing small phase offsets. For example, consider two beams and two cavities. For the lower energy beam, the rf phase of two cavities are $-30^{\circ}$ away from maximum acceleration. For the higher energy beam, first cavity and second cavity phases are set to $-30-d \phi_{1}$ and $-30+d \phi_{2}$, respectively. Depending on the sign of the phase offset $d \phi_{1,2}$ the separation length can be adjusted. The phase optimization must be carried out to satisfy efficient acceleration, proper separation length, and smooth longitudinal envelope. When the separation length is even longer that such remedy cannot be applied, we can make the two beam phase slip by $2 \pi$ not for every single cavity but for every multiple cavities.

Second, the phase synchronization multipass scheme more than twice is not yet realized. Although Ref. [8] proposed a phase shifter between every cavity, it is not yet studied in detail. If we allow different phase offsets for different beams, it might be realizable with fewer phase shifters. It is a future research topic in our study plan.

Third, depending on the time structure of the beam, the high energy beam can catch up to the low energy beam and collide. Since the beam size is much larger compared to the beam size at the interaction points of the collider, the collision effect is expected to be small. A possible issue might be beam loading if two beams collide inside of a cavity. But, it can be avoided by shaping the time structure or introducing drift space at interaction points. For example, in case of Project-X, one bucket is filled out of four for the continuous wave operation [11]. Since the phase slippage between two beams is $2 \pi$ every single cavity, one can replace every fourth cavity by drift space.

\section{ACKNOWLEDGMENTS}

This work was supported by the U.S. Department of Energy under Contract No. DE-AC02-05CH11231 and used computer resources at the National Energy Research Scientific Computing Center.

[1] H. Äit Abderrahim et al., Report Nos. FERMILAB-FN0907-DI, LA-UR-10-06754, 2010.

[2] W. Chou, Report No. FERMILAB-CONF-06-213-AD, 2006.

[3] J. Auerhammer, H. Genz, H.-D. Gräf, R. Hahn, P. Hoffmann-Stascheck, C. Lttge, U. Nething, K. Rühl, A. Richter, T. Rietdorf, P. Schardt, E. Spamer, F. Thomas, O. Titze, J. Töpper, and H. Weise, The S-DALINAC facility-operational experience from the accelerator and 
the experimental installations, Nucl. Phys. A553, 841 (1993).

[4] C. W. Leemann, D. R. Douglas, and G. A. Krafft, The continuous electron beam accelerator facility: CEBAF at the Jefferson Laboratory, Annu. Rev. Nucl. Part. Sci. 51, 413 (2001).

[5] J. Corlett, W. Barletta, S. DeSantis, L. Doolittle, W. Fawley, M. Green, P. Heimann, S. Leone, S. Lidia, D. Li, A. Ratti, K. Robinson, R. Schoenlein, J. Staples, W. Wan, R. Wells, A. Wolski, A. Zholents, F. Parmigiani, M. Placidi, W. Pirkl, R. Rimmer, and S. Wang, A recirculating linac-based facility for ultrafast $\mathrm{X}$-ray science, in Proceedings of the IEEE Particle Accelerator Conference, edited by J. Chew, P. Lucas, and S. Webber (IEEE, New York, 2003), Vol. 1, pp. 186-188.

[6] S. A. Bogacz, Recirculating linac accelerationâ end to end simulation, AIP Conf. Proc. 1222, 363 (2010).

[7] P. H. Williams, D. Angal-Kalinin, D. J. Dunning, J. K. Jones, and N.R. Thompson, Recirculating linac freeelectron laser driver, Phys. Rev. ST Accel. Beams 14, 050704 (2011).

[8] J. Qiang, Wide energy bandwidth superconducting accelerating cavities, Nucl. Instrum. Methods Phys. Res., Sect. A 795, 77 (2015).
[9] V. N. Litvinenko, R. Hajima, and D. Kayran, Merger designs for ERLs, Nucl. Instrum. Methods Phys. Res., Sect. A 557, 165 (2006).

[10] The rf design phase of the second-pass beam can be different from that of the first-pass beam.

[11] S. D. Holmes et al., Project X: Accelerator Reference Design, arXiv: 1306.5022.

[12] J. Qiang and R. W. Garnett, Smooth approximation with acceleration in an alternating phase focusing superconducting linac, Nucl. Instrum. Methods Phys. Res., Sect. A 496, 33 (2003).

[13] J. Qiang, R. D. Ryne, S. Habib, and V. Decyk, An objectoriented parallel particle-in-cell code for beam dynamics simulation in linear accelerators, J. Comp. Physiol. 163, 434 (2000).

[14] R. Storn and K. Price, Differential evolution-a simple and efficient heuristic for global optimization over continuous spaces, J. Global Optim. 11, 341 (1997).

[15] J. Qiang, P. L. Colestock, D. Gilpatrick, H. V. Smith, T. P. Wangler, and M.E. Schulze, Macroparticle simulation studies of a proton beam halo experiment, Phys. Rev. ST Accel. Beams 5, 124201 (2002).

[16] S. Y. Lee, Accelerator Physics, 3rd ed. (World Scientific, Singapore, 2012), p. 55. 